\title{
Determinación del nivel de dosis del ácido valproico e influencia de los fármacos inductores y no inductores enzimáticos en pacientes voluntarios de la ciudad de Mérida, Venezuela
}

\author{
Juan Camilo Cotuá Urzola ${ }^{1, a}$, Alexis Morales Ortiz²,a, Maryori Delgado Nilo3,a , Ana María Muñoz Jáuregui ${ }^{4}$, Luis Quiñones \\ Sepúlveda ${ }^{5}$, Alberto Salazar-Granara ${ }^{6}$, Ángel Alvarado-Yarasca ${ }^{7}$
}

\section{RESUMEN}

Objetivo: Determinar los niveles/dosis (ND) del ácido valproico y la influencia de los fármacos inductores y no inductores enzimáticos en pacientes voluntarios de la ciudad de Mérida-Venezuela.

Materiales y métodos: Se realizó un estudio experimental, observacional, prospectivo de corte transversal, en el Hospital de Mérida-Venezuela. Luego de pasar por los criterios de inclusión y exclusión, los pacientes firmaron el consentimiento informado en forma individual y voluntaria. El protocolo consistió en obtener la sangre de 88 pacientes (42 hombres y 46 mujeres) con edades entre 16 y 68 años, que sufrían de convulsiones parciales o generalizadas y que estaban recibiendo 3,97-38,04 mg/kg de ácido valproico (AVP) dos veces al día en monoterapia o en combinación con carbamacepina (CBZ), fenobarbital (PB), fenitoína (PHT), lamotrigina (LTG) y oxcarbacepina (OXC). Se tomó la muestra después de cuatro semanas de tratamiento, en condiciones de ayuno y antes de la administración del medicamento del presente día, para determinar posteriormente la concentración plasmática por medio del método de radioinmunoensayo. Con los datos de la concentración plasmática se determinó el ND.

Resultados: En monoterapia con AVP se encontró que la concentración plasmática media era de 64,94 mg/l (DE 31,7) y el ND de 4,32 (DE 2,1). En combinación de AVP+CBZ+PB se obtuvo un ND de 2,31 (DE 0,06), con la combinación AVP+PB+PHT se obtuvo el ND de 2,46 (DE 0,43) y con la combinación AVP+PB+OXC se obtuvo un ND de 4,63 (DE 3,12).

Conclusiones: Los fármacos administrados en forma concomitante influyen sobre la concentración plasmática y el ND del AVP en un grupo de pacientes de Mérida-Venezuela.

Palabras clave: Nivel-dosis (ND); ácido valproico; fármacos inductores; fármacos no inductores (Fuente: DeSC BIREME).

\section{Determination of valproic acid dose level and influence of enzyme-inducing and non-enzyme-inducing drugs in volunteer patients of the city of Mérida, Venezuela}

\section{ABSTRACT}

Objective: To determine valproic acid level/dose ratio (ND) and the influence of enzyme-inducing and non-enzymeinducing drugs in volunteer patients of the city of Mérida, Venezuela.

Materials and methods: An experimental, observational, prospective, cross-sectional study was conducted at the Hospital de Mérida, Venezuela. After undergoing the inclusion and exclusion criteria process, patients signed an informed consent individually and voluntarily. The protocol consisted in collecting blood from 88 patients (42 males y 46 females) from 16 to 68 years old, who had partial or generalized seizures, and were receiving 3.97-38.04 mg/kg of valproic acid (AVP) twice a day as monotherapy or combination therapy with carbamazepine (CBZ), phenobarbital (PB), phenytoin (PHT), lamotrigine (LTG) and oxcarbazepine (OXC). A sample was taken after four weeks of treatment, in the fasting state and before the administration of the medication of the day. Afterwards, plasma concentration was determined through a radioimmunoassay method. The ND was established using the plasma concentration data.

Results: AVP monotherapy showed a mean plasma concentration of $64.94 \mathrm{mg} / \mathrm{l}$ (SD 31.7) and ND of 4.32 (SD 2.1). Combination therapy with $\mathrm{AVP}+\mathrm{CBZ}+\mathrm{PB}$ showed an ND of 2.31 (SD 0.06), combination therapy with $\mathrm{AVP}+\mathrm{PB}+\mathrm{PHT}$ showed an ND of 2.46 (SD 0.43) and combination therapy with AVP+PB+OXC showed an ND of 4.63 (SD 3.12).

Conclusions: The administration of concomitant drugs affected plasma concentration and AVP ND in a group of patients of the Hospital de Mérida, Venezuela.

Keywords: Level/dose ratio (ND); valproic acid; enzyme-inducing drugs; non-enzyme-inducing drugs (Source: MeSH NLM).

1. Médico Cirujano, Neurólogo.

2. Magíster en Ciencias, Toxicólogo, UNITOXFAR.

3. Médico Cirujano, Neurólogo.

4. Doctora en Farmacia y Bioquímica, Facultad de Ciencias de la Salud, Universidad San Ignacio de Loyola. Lima,Perú.

5. Doctor en Ciencias Biomédicas, Laboratorio de Carcinogénesis Química y Farmacogenética, Facultad de Medicina. Universidad de Chile.

6. Doctor en Medicina, Unidad de Bioequivalencia y Medicina Personalizada, Facultad de Medicina Humana de la Universidad de San Martín de Porres. Lima, Perú.

7. Magíster en Ciencias Básicas Médicas: Farmacólogo, Departamento de Biología y Química, Facultad de Ingeniería, Universidad San Ignacio de Loyola. Lima, Perú.

a. Universidad de Los Andes Mérida. Venezuela. 
Determinación del nivel de dosis del ácido valproico e influencia de los fármacos inductores y no inductores enzimáticos en pacientes voluntarios de la ciudad de Mérida, Venezuela

\section{INTRODUCCIÓN}

La Medicina Personalizada (MP) implica el tratamiento y prevención de las enfermedades, en el momento correcto, con la dosis correcta y con el paciente correcto, basado en la variabilidad genética individual, el ambiente, el mestizaje, la etnia, el estilo de vida, en la Farmacogenética y en la Farmacocinética Clínica ${ }^{(1,2,3,4)}$. Para promover la MP se debe contar con medicamentos multifuentes bioequivalentes e intercambiables, que garanticen una concentración plasmática dentro del margen terapéutico ${ }^{(5,6)}$. Mediante la Farmacocinética Clínica se realiza la monitorización de los fármacos en plasma, garantizando que alcance la concentración plasmática en el estado estacionario (Css), y mediante la Farmacogenética se estudia el genotipo para determinar el fenotipo metabólico de lento, extensivo y rápido.

La monitorización de concentraciones de fármacos en plasma, es un sistema de Seguimiento Farmacoterapéutico que permite realizar los cambios necesarios según la evolución clínica del paciente y en forma individualizada (7). Mantener los niveles plasmáticos de los fármacos dentro del margen terapéutico, permite minimizar las reacciones adversas y limitar las interacciones farmacológicas; adicionalmente, la monitorización se realiza para los fármacos de estrecho margen terapéutico, para fármacos que se biotransforman en metabolitos activos, en caso de una buena correlación entre las concentraciones plasmáticas y el efecto terapéutico o tóxico, en caso de dificultad para reconocer el efecto farmacológico o tóxico y en caso de una relación dosis-concentración muy variable. Las concentraciones plasmáticas de un fármaco dependen de las dosis administradas, del volumen aparente de distribución $(\mathrm{Vd})$ y de la depuración, que condicionan el tiempo de vida media de eliminación (t1/2) y, por tanto, el tiempo necesario para alcanzar la concentración en el estado estacionario, y es en este momento cuando se recomienda hacer la primera determinación ${ }^{(7)}$.

Los anticonvulsivantes como el ácido valproico, carbamacepina, etosuximida, fenitoína y el fenobarbital, son fármacos de estrecho margen terapéutico, que deben ser monitorizados para garantizar su seguridad y eficacia ${ }^{(7)}$.

El ácido valproico (ácido 2-propilpentanoico) es un ácido graso simple ramificado ${ }^{(8,9)}$, con una masa molar de 144,21 $\mathrm{g} / \mathrm{mol}^{(9)}$, que se absorbe rápidamente después de una dosis oral, alcanzando una concentración plasmática máxima de (Cmáx) 23,5-25,3 mg/l, con un tiempo máximo (tmáx) de $1,5^{(10)}-16,4$ horas, con un $A B C 0-\infty$ de $626-831 \mathrm{mg} . \mathrm{h} / \mathrm{l}$ ${ }^{(11,12)}$ y un pKa de $4,8^{(10)}$. En el adulto la unión a proteínas plasmáticas (UP) es de $85-95 \%^{(10,13)}$, en los ancianos es de un $80-90 \%{ }^{(10)}$, con un $\mathrm{t} 1 / 2$ de $5-20$ horas $(13,14)$, mientras que la sal de valproato de sodio tiene un $\mathrm{t} 1 / 2$ de 7-12,7 horas $^{(12,15)}$; siendo su Vd en el adulto de $0,10-0,19 \mathrm{l} / \mathrm{kg}$ y en el niño de $0,20-0,30 \mathrm{l} / \mathrm{kg}^{(10,13)}$. Se metaboliza por fase I de hidroxilación con participación de la CYP2C9 ${ }^{(16,17)}$, CYP2C19 ${ }^{(16,18)}$, y por la CYP2A6B ${ }^{(16,17)}$. Se tiene dos variantes del gen CYP2C9, de importancia clínica, como el CYP2C9*2 con una actividad enzimática del 4-6\% y el CYP2C9*6 con una actividad nula ${ }^{(19)}$. El metabolito 3-hidroxivalproico, se conjuga por fase II mediante glucuronidación, en O-Bglucurónido de valproato, con participación de UGT1A3, UGT1A4, UGT1A6, UGT1A8, UGT1A9, UGT1A10, UGT2B7 y UGT2B15 ${ }^{(20)}$.

Es considerado un inhibidor enzimático de la CYP2C9, de la epóxido hidroxilasa que metaboliza a la carbamacepina, y de la UGT2B15 que metaboliza lamotrigina (20); recientemente se ha reportado que en la rata es un inductor de su propia glucuronidación, in vitro es un inductor de CYP3A4 y de la glicoproteína-P; y en estudios clínicos ha demostrado efectos inductivos clínicamente relevantes sobre la olanzapina, clozapina, aripiprazol e irinotecan ${ }^{(14)}$.

Siendo la concentración plasmática mínima efectiva $(\mathrm{CmE})$ de $50 \mathrm{mg} / \mathrm{l}$ (347 mol/l) y la concentración plasmática máxima efectiva (CME) de $100 \mathrm{mg} / \mathrm{l}$ (693 mol/l), por encima de la cual se asocia a las reacciones adversas y a la neurotoxicidad ${ }^{(13,16,21)}$. Se menciona que existe una escasa correlación entre la dosis administrada y las concentraciones séricas del ácido valproico debido a su elevada unión a las proteínas plasmáticas, por lo que los cambios en la fracción libre del fármaco no son proporcionales al aumento de la dosis ${ }^{(16)}$. Hermida y Tutor proponen en pacientes con hipoalbuminemia el empleo de una ecuación para determinar la fracción libre del ácido valproico $(\mathrm{H})$ : $\mathrm{Ha}=$ Concentración libre $(\mathrm{CF})$ / Concentración total del fármaco $(\mathrm{CH})^{(22)}$. Hay medicamentos que desplazan al valproato de su unión proteica, lo que lleva a un aumento de la fracción libre del fármaco e incluso un cambio de las vías metabólicas del valproato ${ }^{(9)}$.

El objetivo de este estudio fue determinar los niveles/dosis (ND) del ácido valproico y la influencia de los fármacos inductores y no inductores enzimáticos en pacientes voluntarios de la ciudad de Mérida-Venezuela.

\section{MATERIALES Y MÉTODOS}

\section{Materiales y equipos}

Los materiales utilizados fueron las cubetas Tencell, tubos para muestras con capacidad de $2 \mathrm{ml}$ y micropipetas automáticas; entre los reactivos utilizados fueron la solución de lavado al 4,5\%, Kit de fármacos, controles TDM nivel, 1,2 y 3; entre los equipos utilizados fueron, una centrifuga modelo PLC-05 y un equipo Indiko Thermo Scientific.

\section{Tipo y diseño de investigación}

Se realizó un estudio experimental, observacional, prospectivo de corte transversal, en el Hospital de MéridaVenezuela.

\section{Muestras de estudio}

Se obtuvo la sangre de 88 pacientes voluntarios con edades que comprendían entre 16 y 68 años (media 36,43, DE 14), que sufrían de convulsiones parciales o generalizadas y que estaban recibiendo ácido valproico (AVP) dos veces al día 
en monoterapia de 3,97-38,04 mg/kg (media 16,69, DE 8,4) o en combinación con otros fármacos antiepilépticos, tales como carbamacepina (CBZ), fenobarbital (PB), fenitoína (PHT), lamotrigina (LTG) y oxcarbacepina (OXC) ${ }^{(23)}$.

\section{Consentimiento informado}

Los pacientes firmaron el consentimiento informado en forma individual y voluntaria, luego de escuchar las explicaciones sobre los objetivos, la importancia y la aplicación del estudio en la salud pública.

\section{Criterios de inclusión y exclusión}

El médico especialista tratante explicó sobre los alimentos, tipos bebidas y medicamentos que deberían consumir una semana antes del estudio; y a la vez, estar en ayunas el día de la toma de la muestra. Antes de la toma de la muestra, se interrogó a todos los pacientes voluntarios, sobre los criterios de cumplimiento y de ayuno; siendo excluidos todos aquellos que no cumplieron con dichos criterios. Se excluyeron del estudio a los pacientes con insuficiencia renal y con disfunción hepática ${ }^{(23)}$.

\section{Aspectos éticos}

Los investigadores del presente estudio garantizan la confidencialidad y el anonimato de los datos de los pacientes voluntarios. La información obtenida solo se utilizará con fines académicos y científicos.

\section{Técnica y metodología de la extracción de la muestra biológica}

Las muestras de sangre se colectaron como parte de la rutina del tratamiento y del seguimiento farmacoterapéutico.
Todas las muestras sanguíneas se colectaron en tubos BD Vacutainer de $3 \mathrm{ml}$, el mismo que se realizó después de cuatro semanas de tratamiento (más de 4 tiempos de vida media), es decir, luego de alcanzar la concentración plasmática en el estado estacionario (Css), en condiciones de ayuno, entre las 8:00-10:00 am, y con no menos de 10 horas después de la última toma de la dosis y antes de la administración del medicamento del presente día ${ }^{(16,23,24)}$.

\section{Método de cuantificación}

Para la cuantificación de los fármacos en plasma, primero se centrifugó $2 \mathrm{ml}$ de sangre de cada paciente a $8000 \mathrm{rpm}$ por $10 \mathrm{~min}$, luego se midió 500 microlitros de suero y se analizaron por duplicado por el método de radioinmunoensayo en el equipo Indiko Thermo Scientific (24).

\section{Técnica de análisis de datos}

El análisis se realizó mediante la estadística descriptiva como la distribución de frecuencias. Dichos resultados se registraron en un instrumento de colección de datos, posteriormente se tabuló en una base de datos para analizarse con el programa informático Statistical Package for Social Sciences (SPSS) versión 20,0 para Windows.

\section{RESULTADOS}

En la tabla 1 se presenta la estadística descriptiva de las variables cuantitativas y su relación entre la dosis con la concentración plasmática y la relación nivel/dosis del ácido valproico.

Tabla 1. Relación entre la dosis, la concentración y el nivel/dosis del ácido valproico

\begin{tabular}{|c|c|c|c|c|c|c|c|}
\hline Variables & Media & Mediana & Moda & DE & Rango & Mínimo & Máximo \\
\hline Edad & 36,43 & 36,00 & 16 & 14,0 & 52 & 16 & 68 \\
\hline Peso & 69,28 & 67,450 & 64,0 & 17,1 & 86,0 & 40,0 & 126,0 \\
\hline $\begin{array}{l}\text { Dosis de AVP } \\
(\mathrm{mg} / \mathrm{Kg})\end{array}$ & 16,69 & 13,99 & 25,30 & 8,4 & 34,07 & 3,97 & 38,04 \\
\hline $\begin{array}{l}\text { Concentración } \\
\text { plasmática } \\
\text { (mg/l) }\end{array}$ & 64,94 & 59,35 & 33,20 & 31,7 & 150,40 & 5,60 & 156,00 \\
\hline $\begin{array}{l}\text { Nivel/dosis } \\
\text { (N/D) }\end{array}$ & 4,32 & 4,03 & 2,99 & 2,1 & 11,41 & 0,59 & 12,00 \\
\hline
\end{tabular}

En la tabla 2 se presenta los resultados de la t de Student para muestras independientes: promedio de la relación nivel/dosis del ácido valproico con respecto al momento de la toma de la muestra y la respuesta terapéutica del ácido valproico (AVP) que se administraron a los pacientes voluntarios. 
Determinación del nivel de dosis del ácido valproico e influencia de los fármacos inductores y no inductores enzimáticos en pacientes voluntarios de la ciudad de Mérida, Venezuela

Tabla 2. Promedio de la relación nivel dosis del ácido valproico con respecto al momento de la toma de la muestra

\begin{tabular}{lcccc}
\multicolumn{1}{c}{ Variable } & Tipo & Media & DE & $p$ \\
Momento de la toma de muestra & Predosis & 4,32 & 1,71 & 0,000 \\
\cline { 2 - 4 } & Posdosis & 10,45 & 1,94 & 0,20 \\
Respuesta terapéutica & Controlado & 4,60 & 2,82 & 2,56
\end{tabular}

En la tabla 3 se presenta el promedio y desviación estándar del resultado de la relación nivel dosis del ácido valproico administrado en forma concomitante con fármacos anticonvulsivantes inductores y no inductores enzimáticos.

Tabla 3. Resultados de la relación nivel dosis del ácido valproico administrado en forma concomitante con fármacos anticonvulsivantes inductores y no inductores enzimáticos

\begin{tabular}{|c|c|c|c|c|}
\hline $\begin{array}{c}\text { Esquema de } \\
\text { administración }\end{array}$ & Media & $\mathbf{n}$ & DE & $\begin{array}{c}\text { Influencia de } \\
\text { los fármaco } \\
\text { concomitante (\%) }\end{array}$ \\
\hline$A V P+C B Z+P B$ & 2,31 & 2 & 0,06 & 46,53 \\
\hline$A V P+P B+P H T$ & 2,46 & 2 & 0,43 & 43,06 \\
\hline$A V P+P B+L T G$ & 1,41 & 1 & & \\
\hline$A V P+P B+O X C$ & 4,63 & 2 & 3,12 & 7,18 \\
\hline Ninguno & 4,45 & 81 & 2,17 & \\
\hline Total & 4,32 & 88 & 2,18 & \\
\hline
\end{tabular}

\section{DISCUSIÓN}

Concentración plasmática y nivel dosis del ácido valproico

En nuestro estudio hemos encontrado que la concentración plasmática media del ácido valproico es de 64,94 mg/l (DE 31,7 ), con un nivel/dosis (N/D) de 4,32 (DE 2,1), obtenidos con una dosis media de $16,69 \mathrm{mg} / \mathrm{kg}(\mathrm{DE} 8,4)$ y en pacientes ambulatorios, de los cuales 42 fueron hombres $(47,73 \%)$ y 46 fueron mujeres (52,27\%). Nuestros resultados se encuentran dentro del intervalo de referencia que fue reportado por Aldaz et al, el mismo que es de 50-100 mg/l (347-693 $\mu \mathrm{mol} / \mathrm{l})$ (16), valores superiores a la concentración plasmática máxima efectiva (CME) $175 \mathrm{mg} / \mathrm{l}$ se asocian con un riesgo elevado de neurotoxicidad, y con valores por debajo de la concentración plasmática mínima efectiva (CmE $50 \mathrm{mg} / \mathrm{l})$ hay un fracaso en la terapia farmacológica.

Además, se encontró que la relación nivel/dosis es muy significativa ( 0,000$)$ cuando la toma de la muestra se realiza en predosis. Esto se obtiene, ya que el tiempo de extracción óptima para la monitorización de los tratamientos antiepilépticos, administrados por vía oral, se realizó de acuerdo a lo propuesto por Aldaz et al, es decir, justo antes de la dosis matinal del fármaco, cuya concentración plasmática obtenida se denomina concentración valle, basal, mínima o predosis ${ }^{(16)}$.Esta estrategia de muestreo es importante ya que permite hacer los estudios y la monitorización de los fármacos en la práctica clínica, para proponer la dosis correcta y en el momento correcto.

\section{Influencia de los fármacos anticonvulsivantes concomitantes}

Los pacientes que recibieron $\mathrm{AVP}$ en combinación con inductores enzimáticos, como $\mathrm{CBZ}+\mathrm{PB}$, mostraron una relación ND media del 46,53\% menor $(2,31 ; n=2 ; D E=0,06)$, mientras que la combinación de $\mathrm{PB}+\mathrm{PHT}$, redujeron un 
$43,06 \%$ la relación ND media del AVP $(2,46 ; n=2 ; D E=0,43)$; en ambos casos en comparación con la monoterapia (ND $4,32 ; \mathrm{DE}=2,1)$. Además, nuestros resultados indican que la influencia de los fármacos inductores es similar en hombres y mujeres. En Latinoamérica no se tiene estudios sobre el ND, pero, si podemos correlacionar nuestros resultados, con el de Adín et al, quienes reportaron que el nivel/dosis del topiramato (TMP) con inductores enzimáticos, como CBZ, PB y $\mathrm{PHT}$, se reduce a un $60 \%$, en comparación de quienes recibieron TMP con no inhibidores como el AVP o LTG ${ }^{(23)}$.

El ND es un método que nos permite cuantificar y expresar el grado de inducción de los fármacos concomitantes y que puede ser de utilidad clínica para realizar la medicina personalizada. Sin embargo, debemos manifestar que hay modelos farmacocinéticos que analizan los factores que influyen en el aclaramiento del fármaco, obteniendo una dosis con mayor precisión, tal es el caso de Nakashima et al quienes establecieron una dosis diaria de AVP para mantener dentro del margen terapéutico la concentración plasmática del fármaco $(50-100 \mathrm{mg} / \mathrm{l})$, para ello, se consideró los factores que influyen en el aclaramiento del AVP, tales como la edad del paciente, el sexo, el peso corporal y la coadministración de CBZ, PHT, PB y/o clobazam (CLB); aunque algunos autores no han encontrado una alta correlación entre las concentraciones plasmáticas de AVP y los efectos terapéuticos o tóxicos del fármaco ${ }^{(25)}$. En otro estudio multicéntrico realizado por Lin et al se estableció mediante el modelo farmacocinético en pacientes adultos epilépticos de China que la dosis de AVP era de 750 mg BID, ya que después de tomar el fármaco por espacio de tres meses, se encontró que la concentración plasmática estaba en $60 \mathrm{mg} / \mathrm{l}$, con la cual las frecuencias de convulsiones disminuyeron gradualmente, y no se tuvo convulsiones durante casi 2 meses, además de mantener la función hepática normal ${ }^{(26)}$. Una influencia significativa es el fenotipo del paciente, tal como lo ha demostrado Büdi et al, quienes reportaron que la CYP2C9 influye sobre la concentración plasmática del AVP, disminuyendo la incidencia de la hiperamonemia, pero no se evitó el cambio de peso y la somnolencia ${ }^{(24)}$.

En pacientes que tuvieron tratamiento de $\mathrm{AVP}+\mathrm{PB}+$ OXC se encontró que hay un predominio de los fármacos no inductores enzimáticos (AVP $+\mathrm{OXC})$ sin predominio del $\mathrm{PB}$, ya que se incrementó la relación ND en un $7,18 \%(4,63 ; n=2 ; D E=3,12)$. Esto tiene una correlación con el estudio de May et al ${ }^{(27)}$ y con el de Aldaz et al ${ }^{(16)}$ quienes reportan que la OXC es un profármaco que se metaboliza independientemente del CYP450 por un proceso de reducción cetónica, en un metabolito activo, la licarbamacepina o 10-hidroxicarbamacepina (MHD), que luego se conjuga con el ácido glucurónico, por este motivo, su aclaramiento plasmático no está influido por la administración concomitante con otros fármacos inductores o inhibidores.
Sin embargo, la influencia de cada fármaco anticonvulsivante inductor y no inductor encontrada en nuestro estudio puede estar sesgada, debido al número pequeño de la muestra, lo que amerita a seguir investigando con un mayor número de pacientes y podamos tener un ND para los venezolanos.

Para nuestros países de Latinoamérica, es importante determinar el ND, determinar las concentraciones plasmáticas de los fármacos y establecer el fenotipo metabólico de los pacientes, para mejorar la seguridad de las terapias antiepilépticas y promover la implementación de la medicina personalizada.

En conclusión, en base a los resultados obtenidos de la concentración plasmática media $(64,94 \mathrm{mg} / \mathrm{l}$; DE 31,7$)$ y del nivel/dosis del ácido valproico (N/D 4,32; DE 2,1), logrados a una dosis media de $16,69 \mathrm{mg} / \mathrm{kg}(\mathrm{DE} 8,4)$, y luego de la administración concomitante de fármacos inductores enzimáticos ( $C B Z+P B ; P B+P T H)$ y no enzimáticos $(\mathrm{OXC})$, se concluye que dichos fármacos influyen en el ND del AVP en el grupo de pacientes voluntarios y ambulatorios de la ciudad de Mérida-Venezuela.

\section{REFERENCIAS BIBLIOGRÁFICAS}

1. Bravo S, Caminos J, Eslava J. Medicina personalizada: farmacogenómica y farmacoepigenética. Rev Col Anestesiología. 2011; 39(3):308-13.

2. Medrano A. Medicina personalizada: hacia un nuevo modelo en la práctica médica. Arch Neurocien (Mex). 2012; 17(2):129-31.

3. Precision Medicine Initiative (PMI) Working Group Report to the Advisory Committee to the Director, NIH. The Precision Medicine Initiative Cohort Program-Building a Research Foundation for 21st Century Medicine. Estados Unidos, 2015.

4. Garassino M. Medicina personalizada para el cáncer: Una guía ESMO para los pacientes. Sociedad Europea de Oncología Médica. [Internet]. 2013 [citado 25 de abril de 2017]. Recuperadp a partir de: https://www.esmo.org/content/ download/46499/855050/file/ESMO-Medicina-PersonalizadaGuia-para-Pacientes.pdf

5. Alvarado A, Salazar A, Pineda N, Villanueva H, Cáceres E. Estudio de la biodisponibilidad relativa de una formulación multifuente de sulfametoxazol respecto al medicamento referente. Horiz Med. 2016;16 (3):12-9.

6. Alvarado A, Lozada G, Llerena R, Macavilca S, Marcos E, Pisconte N, Poma J, Ismodes Y. Determinación del margen terapéutico y estudio de la equivalencia biofarmacéutica de las tabletas multifuentes de digoxina de 0,25 mg. Horiz Med. 2014;14 (4): 48-52.

7. Fernández E, Serrano D, Villamarín L. Monitorización clínica de medicamentos. Butlletí d'informació Terapéutica 2011;22(9):51-5.

8. RET. Farmacología básica del valproato. Revista de Toxicomanías. [Internet]. 2006 [citado 25 de abril de 2017];47:11-33. Recuperadp a partir de: http://www.catbarcelona.com/uploads/rets/47_2.pdf

9. Gamez G, Zhu L, Disko A, Chen H, Azov V, Chingin K, et al. Real-time, in vivo monitoring and pharmacokinetics of valproic acid via a novel biomarker in exhaled breathw. Chem. Commun. 2011;47: 4884-6.

10. Salas-Puig J. Farmacología del valproato sódico. Emergencias. 2005; 17:79-82.

11. Dutta S, Zhang Y, Lee L, O'Dea R. Comparison of the 
Bioavailability of 250 and 500mg Divalproex Sodium ExtendedRelease Tablets in Healthy Volunteers. Biopharm. Drug Dispos. 2004;25(8): 353-7.

12. Dulac O, Alvarez JC. Bioequivalence of a New SustainedRelease Formulation of Sodium Valproate, Valproate ModifiedRelease Granules, Compared with Existing Sustained-Release Formulations After Once- or Twice-Daily Administration. Pharmacotherapy. 2005;25(1):35-41.

13. Lorenzo P, Moreno A, Leza J, Lizasoain I, Moro M. Velásquez Farmacología Básica y Clínica. Madrid. 17 ed. Mexico. Panamericana; 2005.

14. Lana F, Marti-Bonany J, Fuster J, de León J. Reduction in serum concentration of valproic acid secondary to the intake of ibuprofen as an example of valproico acid auto-induction metabolism. Actas Esp Psiquiatr. 2016;44(4):136-44.

15. Fujisaki Y, Tsukune T, Funyû M, Okumura M, Ukigaya T, Sugibayashi K. Development of Sustained-ReleaseTablets Containing Sodium Valproate: In Vitro and In Vivo Correlation. Drug Development and Industrial Pharmacy. 2006;32(2) :207-17.

16. Aldaz A, Ferriols R, Aumente D, Calvo MV, Farre MR, García $B$, et al. Monitorización farmacocinética de antiepilépticos. Farm Hosp. 2011;35(6):326-39.

17. Sabin O, Pop R, Trifa A, Buzoianu A. The influence of CYP2C9, CYP2C19 and ABCB1 polymorphisms on the plasma concentrations of valproic acid in epileptic patients. Human $y$ Veterinary Medicine International Journal of the Bioflux Society. 2016;8(1):29-33.

18. Komatsu T, Yamazaki H, Asahi S, Gillam E, Guengerich F, Nakajima $M$, et al. Formation of a dihydroxy metabolite of phenytoin in human liver microsomes cytosol: roles of cytochromes P450 2C9, 2C19, and 3A4. Drug Metab Dispos. 2000;28(11):1361-9.

19. Saldaña A, Sánchez J, Márquez D, García A, Flores S. Farmacogenética y metabolismo de fármacos antiepilépticos: implicación de variantes genéticas en citocromos P450. Rev Neurol. 2013; 56 (9):471-9.

20. Ghodke Y, Thorn C, Lamba J, Leeder J, Song W, Birnbaum $A$, et al. Valproic acid pathway: pharmacokinetics and pharmacodynamics. Pharmacogenet Genomics. 2013;23(4): 236-41.

21. Zhao M, Li G, Qiu F, Sun Y, Xu Y, et al. Development and Validation of a Simple and Rapid UPLC-MS Assay for Valproic Acid and Its Comparison With Immunoassay and HPLC Methods. Ther Drug Monit. 2016; 38 (2):246-52.

22. Hermida J, Tutor J. A Theoretical Method for Normalizing Total Serum Valproic Acid Concentration in Hypoalbuminemic Patients. Journal of Pharmacological Sciences. 2005;97(4): 489-93.
23. Adín J, Cruz M, Blanco Y, Herranz J, Armijo J. Topiramate Serum Concentration-to-Dose Ratio:Influence of Age and Concomitant Antiepileptic Drugs and Monitoring Implications. Ther Drug Monit. 2004;26(3):251-7.

24. Büdi T, Tóth K, Nagy A, Szever Z, Kiss A, Temesvári M, et al. Clinical significance of CYP2C9-status guided valproic acid therapy in children. Epilepsia. 2015:56(6):849-55.

25. Nakashima $H$, Oniki K, Nishimura $M$, Ogusu $N$, Shimomasuda $M$, Ono T, et al. Determination of the Optimal Concentration of Valproic Acid in Patients with Epilepsy: A Population Pharmacokinetic-Pharmacodynamic Analysis. Plos one. 2015:10(10); 1-14.

26. Lin W, Jiao Z, Wang Ch, Wang $\mathrm{H}$, Ma Ch, Huang $\mathrm{P}$, et al. Population Pharmacokinetics of Valproic Acid in Adult Chinese Epileptic Patients and its Application in an Individualized Dosage Regimen. Ther Drug Monit. 2015;37(1):76-83.

27. May T, Korn-Merker E, Rambeck B. Clinical pharmacokinetics of oxcarbazepine.Clin Pharmacokinet. 2003;42(12):1023-65.

\section{Fuentes de financiamiento:}

Este artículo ha sido financiado por los autores.

\section{Conflictos de interés:}

Los autores declaran no tener ningún conflicto de interés.

\section{Correspondencia:}

Ángel Tito Alvarado-Yarasca

Dirección: Av. La Fontana 550, La Molina. Lima, Perú.

Teléfono: 964321693

Correo electrónico: eaa.alvarado@hotmail.com

Recibido: 10 de mayo de 2017

Evaluado: 11 de mayo de 2017 Aprobado: 25 de mayo de 2017

(c) La revista. Publicado por Universidad de San Martín de Porres, Perú. (cc) в bajo términos de Licencia Creative Commons Atribución 4.0 Internacional. (http://creativecommons.org/licenses/by/4.0/) 